\title{
AB4 inhibits Notch signaling and promotes cancer cell apoptosis in liver cancer
}

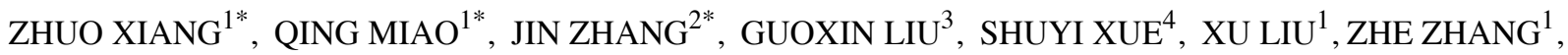 \\ LIXIA SHEN $^{1}$, BANGGUO LIU $^{1}$, YU ZHOU $^{5}$, TING MIAO $^{6}$ and YANG LIU ${ }^{1 *}$ \\ Departments of ${ }^{1}$ Pharmacy, ${ }^{2}$ Burn and Plastic Surgery, Hospital 971 of The Navy of Chinese PLA, Qingdao, \\ Shandong 266071; ${ }^{3}$ Department of Pharmacy, The Third People's Hospital of Qingdao, Qingdao, Shandong 266071; \\ ${ }^{4}$ Department of Pharmacy, Qingdao Central Hospital, Qingdao, Shandong 266071; \\ ${ }^{5}$ Wuxi Center for Drug Safety Control, Wuxi, Jiangsu 214000; ${ }^{6}$ Department of Traditional Chinese Medicine, \\ Hospital 971 of The Navy of Chinese PLA, Qingdao, Shandong 266071, P.R. China
}

Received October 18, 2020; Accepted March 29, 2021

DOI: $10.3892 /$ or.2021.8063

\begin{abstract}
The etiology for liver cancer has been clearly defined. Unfortunately, therapeutic approaches for liver cancer are rather limited, and liver cancer is insensitive to chemotherapy and radiotherapy. Traditional Chinese medicine (TCM) has become a promising strategy for cancer treatment as TCM elicits broad spectrum anticancer activity. In the present study, we evaluated the anticancer efficacy of AB4, an extract from the medical herb Pulsatilla chinensis (Bunge) Regel, in liver cancer in vitro and in vivo. We found that $\mathrm{AB} 4$ readily doseand time-dependently inhibited liver cancer HepG2 and Huh-7 cell proliferation and colony formation. Western blot and flow cytometry analyses suggested that AB4 treatment induced liver cancer cell apoptosis. Moreover, these findings could be readily recaptured in vivo, in which the $\mathrm{AB} 4$ regimen resulted in tumor suppression and cancer cell apoptosis in xenograft tumor-bearing nude mice. Importantly, we noted that treatment with a Notch signaling inhibitor DAPT produced very similar anticancer efficacy in both HepG2 and Huh-7 cell lines, and administration of DAPT also efficiently suppressed HepG2 xenograft outgrowth. To this end, we anticipated that AB4 and DAPT may act on the same signaling pathway, probably through inhibition of the Notch pathway. Indeed, we found decreased expression of Notch1 protein, as well as downstream targets Hes1 and Hey1, after AB4 treatment.
\end{abstract}

Correspondence to: Dr Qing Miao or Dr Yang Liu, Department of Pharmacy, Hospital 971 of The Navy of Chinese PLA, 22 Minjiang Road, Qingdao, Shandong 266071, P.R. China

E-mail: miaoqing971@163.com

E-mail: jusesy@163.com

*Contributed equally

Key words: liver cancer, Pulsatilla chinensis (Bunge) Regel, AB4, apoptosis, Notch
Immunohistochemistry analysis further confirmed the suppression of Notch signaling in HepG2 xenograft-bearing mice. Taken together, our study highlighted the anticancer efficacy of AB4 in liver cancer. We also provided preliminary data showing Notch as a therapeutic target of AB4. It would be interesting to investigate the anticancer efficacy of AB4 in other types of cancer with elevated Notch activity.

\section{Introduction}

Liver cancer is one of the leading malignancies worldwide. The etiology of liver cancer varies, which makes the diagnosis and therapeutic strategy a significant challenge. For example, hepatitis B virus infection is the prevalent cause of liver cancer in China and South Africa, whereas $>70 \%$ of liver cancer cases in Japan and the US are associated with chronic infection with hepatitis $\mathrm{C}$ virus (1). Although numerous clinical trials and translational studies have increased the understanding of the molecular mechanisms that drive the initiation and progression of liver cancer, the prognosis of advanced liver cancer remains poor, and novel therapeutics are urgently required (2). For this purpose, traditional Chinese medicine (TCM) has attracted increasing interest as Chinese medicinal herbs can target multiple signaling pathways that are involved in cancer cell survival and proliferation $(3,4)$. Additionally, TCM facilitates conventional chemotherapy to promote liver cancer cell apoptosis and reduce dose-related cytotoxicity of chemotherapeutics (5). Therefore, TCM deserves further investigation as an optimal strategy for advanced liver cancer.

The Chinese medicinal herb Pulsatilla chinensis (Bunge) Regel and its derivates exert various bioactivities, including anti-inflammatory (6), anti-oxidative (7), anti-bacterial and anti-fungal effects (8). Notably, Pulsatilla chinensis (Bunge) Regel extracts have been demonstrated to inhibit cancer cell proliferation in vitro and in vivo, including in A549 lung cancer cells (9), SK-MEL-2 melanoma cells $(10,11)$ and MCF-7 breast cancer cells $(12,13)$. These results have prompted innovative research investigating the isolation of biologically active components of this herb. Previous studies have suggested that 
Pulsatilla saponin A is one of the anticancer components, which kills cancer cells through the induction of cell cycle arrest and apoptosis $(14,15)$. However, Pulsatilla saponin A exhibits severe hemolytic toxicity, which could be a major obstacle for its clinical application as an anticancer agent (16). Anemoside B4 (AB4) is another major component of Pulsatilla chinensis (Bunge) Regel extracts. However, to the best of our knowledge, its biological activity is largely unknown. AB4 is well tolerated and exhibits minimal dose-related cytotoxicity. Therefore, deciphering its pharmacological potential could be a promising strategy for the treatment of patients with advanced liver cancer.

Notch signaling was initially noticed due to the appearance of a notch in the wings of fruit flies, and was subsequently found to serve a critical role in embryonic development. Activation of Notch signaling by its ligand leads to Notch intracellular domain (NICD) release and nucleus translocation, where NICD cooperates with the DNA-binding protein CSL to form a transcriptional active heterodimer, which activates Notch-targeted gene expression (17). Notably, aberrant activation of Notch has been detected in multiple malignancies, and further in-depth laboratory investigations have demonstrated that activation of Notch signaling not only directly leads to tumorigenesis, but also cross-talks with numerous other pathways implicated in tumor invasion, migration and metastasis (18). For example, transgenic mice carrying liver-specific constitutively activated Notch develop liver cancer once they reach adult age, and upregulation of Notch1 expression is an unfavorable prognostic biomarker for patients with liver cancer (18). Notch1 activation contributes to liver cancer cell growth and proliferation, whereas Notch1 downregulation inhibits invasion and migration by inactivating the cyclooxygenase-2/Snail/E-cadherin signaling pathway or via its interaction with PTEN and focal adhesion kinase (19). These data indicate that Notch is a potent therapeutic target for liver cancer. In agreement with this notion, several ongoing phase I/II trials are evaluating the safety and efficacy of Notch inhibitors in multiple solid tumors (20-22). The $\gamma$-secretase inhibitors (GSIs) have shown anticancer efficacy in multiple Notch-mutant solid tumors, while the dose-related cytotoxicity of GSIs largely limits their clinical application (23). Therefore, increasing efforts have been made to develop novel Notch inhibitors (24).

The present study reported the anticancer efficacy of AB4 in liver cancer in vitro and in vivo. AB4 inhibited liver cancer cell proliferation and induced cell apoptosis in a time- and dose-dependent manner. Further mechanical experiments demonstrated that inhibition of Notch signaling might be implicated in the anticancer efficacy of AB4. Overall, the present study highlighted the anticancer activity of the medicinal herb extract $\mathrm{AB} 4$, and targeting of Notch signaling by $\mathrm{AB} 4$ is a potential therapeutic approach for advanced liver cancer.

\section{Materials and methods}

Reagents. AB4 was purchased from Absin Biotechnology Co., Ltd. FBS, DMEM, trypsin and penicillin-streptomycin solution were purchased from HyClone; Cytiva. Primary antibodies against cleaved caspase-3 (\#9661), cleaved poly(ADP-ribose) polymerase (PARP) (\#5625), cytochrome $c$ (\#4280), Notch1 (\#4380), Jagged1 (\#2620), NICD1 (\#4380), hes family bHLH transcription factor 1 (Hes1) (\#11988), , Ki67 (\#9027) and $\beta$-actin (\#4967), and HRP-labeled secondary anti-rabbit IgG
(\#7074) were obtained from Cell Signaling Technology, Inc. The hes related family bHLH transcription factor with YRPW motif 1 (Heyl) antibody (\#ab11723) was from Abcam. The Annexin V-FITC Apoptosis Detection kit was obtained from EMD Millipore. DAPT, a $\gamma$-secretase inhibitor (GSI) that inhibits the Notch pathway, MTT and DMSO were purchased from Sigma-Aldrich; Merck KGaA. The 3,3'-diaminobenzidine reagent was purchased from Dako; Agilent Technologies, Inc.

Cell culture. The hepatoblastoma HepG2 cell line and hepatocellular carcinoma Huh-7 cell line were obtained from The Cell Bank of Type Culture Collection of The Chinese Academy of Sciences. Cell lines were authenticated using short-tandem repeat profiling, tested for mycoplasma contamination and used at passage numbers of $<10$. Cells were maintained in DMEM supplemented with $10 \%$ FBS and $100 \mathrm{U} / \mathrm{ml}$ penicillin-streptomycin solution. Cells were cultured in a humidified incubator with $5 \% \mathrm{CO}_{2}$ at $37^{\circ} \mathrm{C}$.

MTT assay. Cells were seeded into 96-well plates at a density of $5 \times 10^{3}$ cells per well. The cells were cultured overnight to allow attachment, and then the cells were treated with increasing concentrations of AB4 or DAPT (50 $\mu \mathrm{mol} / \mathrm{l})$ for different time periods. Cells were subsequently incubated with $0.5 \mathrm{mg} / \mathrm{ml} \mathrm{MTT}$ for another $4 \mathrm{~h}$. The formazan was dissolved in $100 \mu 1 \mathrm{DMSO}$ and the optical density at $570 \mathrm{~nm}$ was determined using a micro-array reader (Bio-Rad Laboratories, Inc.). Each experiment was performed in triplicate and the data are presented as the mean $\pm \mathrm{SD}$.

Colony formation assay. Cells were seeded into 6-well plates at a density of $1 \times 10^{3}$ cells per well. Following overnight incubation to allow cell attachment, the cells were grown in medium containing AB4 (5 and $10 \mu \mathrm{mol} / \mathrm{l})$ or DAPT ( $5 \mu \mathrm{mol} / \mathrm{l})$ for $\sim 14$ days. The medium was refreshed every 3 days. At the end of the experiment, the cell culture medium was discarded and the cells were washed with PBS three times. After being fixed with $4 \%$ paraformaldehyde for $20 \mathrm{~min}$, the colonies were visualized using crystal violet staining under a microscope.

Apoptosis assay. Apoptosis was determined using an Annexin V-FITC Apoptosis Detection kit (EMD Millipore) according to the manufacturer's protocols. Briefly, cells were seeded into 6-well plates at a density of $2.5 \times 10^{5}$ cells per well, and then treated with AB4 $(50 \mu \mathrm{mol} / \mathrm{l})$ and DAPT $(50 \mu \mathrm{mol} / \mathrm{l})$ for $24 \mathrm{~h}$. Cells were resuspended in $95 \mu \mathrm{l}$ Annexin V-FITC binding buffer mixed with $5 \mu \mathrm{l}$ Annexin V-FITC, followed by incubation with $10 \mu \mathrm{l}$ PI. Flow cytometry was performed using a FACS Calibur flow cytometer.

Western blot analysis. After the indicated treatment, cells were harvested and lysed in lysis buffer. Equal amounts of extracted protein samples (10-30 $\mu \mathrm{g})$ were separated by $10 \%$ SDS-PAGE and transferred onto PVDF membranes. The membranes were blocked with 5\% BSA in TBS with $1 \%$ Tween-20 for $1 \mathrm{~h}$, and then incubated with the corresponding primary antibodies (dilution, 1:1,000) overnight at $4^{\circ} \mathrm{C}$. Protein bands were detected using HRP-conjugated secondary antibodies (dilution, 1:5,000) and visualized using enhanced chemiluminescence (Millipore). 
A
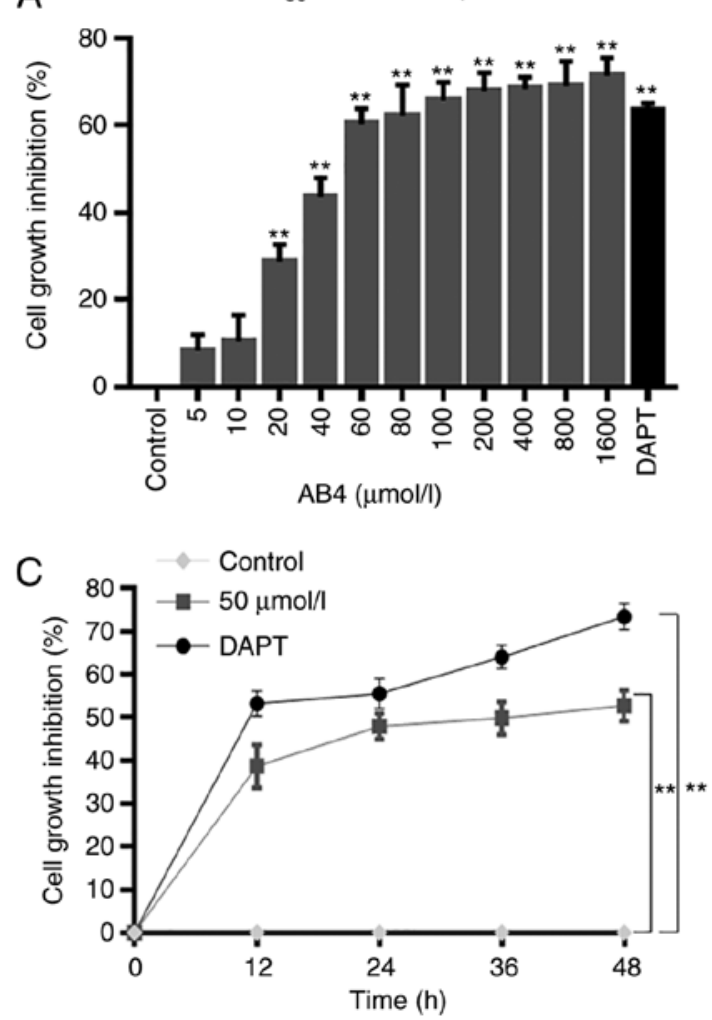

B

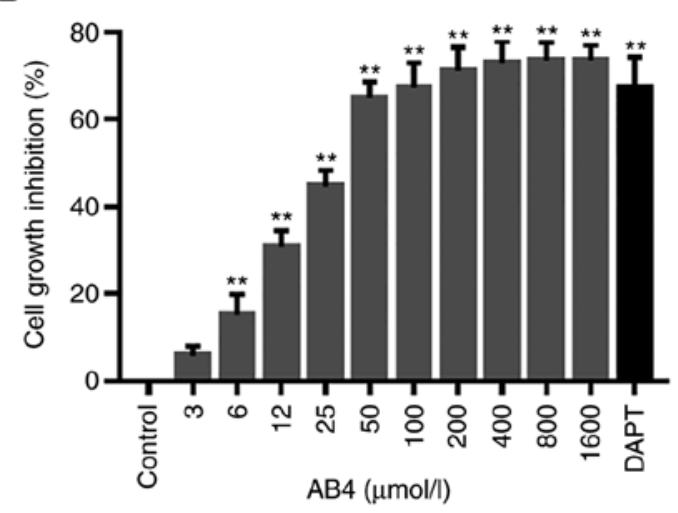

$\mathrm{D}$

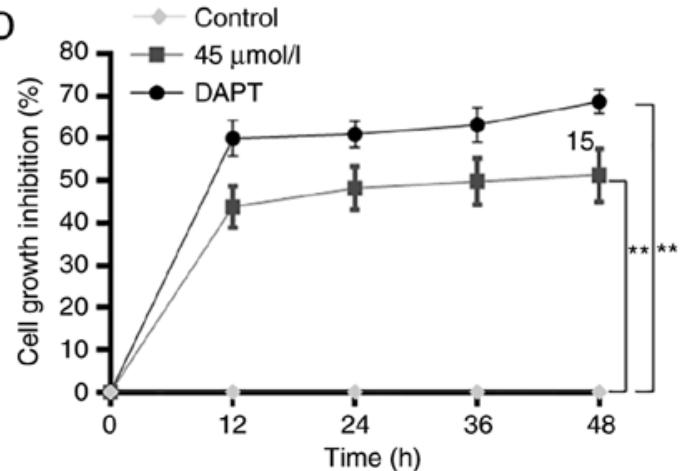

Figure 1. AB4 treatment dose- and time-dependently inhibits liver cancer cell proliferation. (A) HepG2 and (B) Huh-7 cells were treated with the indicated increasing concentrations of $\mathrm{AB} 4$ for $24 \mathrm{~h}$. The inhibitory effect and $\mathrm{IC}_{50}$ of $\mathrm{AB} 4$ on liver cancer cells were measured by MTT assay. (C) HepG2 and (D) Huh-7 cells were treated with $\mathrm{AB} 4$ at a final concentration corresponding to the relevant $\mathrm{IC}_{50}$ value for different time intervals $(0-48 \mathrm{~h})$. The effect of AB4 on liver cancer cell proliferation was determined by MTT assay. Each experiment was performed in triplicate and DAPT was used as a positive control. Data are presented as mean $\pm \mathrm{SD}$. ${ }^{* *} \mathrm{P}<0.01$ vs. vehicle control. AB4, an extract from the medical herb Pulsatilla chinensis (Bunge) Regel; $\mathrm{IC}_{50}$, half maximal inhibitory concentration; DAPT, a $\gamma$-secretase inhibitor (GSI) that inhibits the Notch pathway.

Xenograft tumor model. A total number of 20 specific pathogen-free male nude mice (4 weeks old; $12 \pm 2 \mathrm{~g}$ ) were purchased from Beijing Vital River Laboratory Animal Technology Co., Ltd. The mice were housed in temperature $\left(25^{\circ} \mathrm{C}\right)$ - and humidity $(50 \%)$-controlled feeding rooms under a $12 \mathrm{~h}$ light/dark cycle and provided with unrestricted amounts of rodent chow and drinkable water. All experiments were approved by the Ethic Committee of The Hospital 971 of The Navy of Chinese People's Liberation Army (Qingdao, China) and were performed in accordance with Animal Ethics Guidelines (\#401LL-2017010). HepG2 cells $\left(\sim 2 \times 10^{6}\right)$ were resuspended in $0.2 \mathrm{ml}$ DMEM and subcutaneously injected into the right flank of the nude mice. When the xenograft tumor volume reached $150 \mathrm{~mm}^{3}$, the tumor-bearing mice were randomly divided into four groups ( $\mathrm{n}=5$ in each group), which received vehicle as a control, low dose AB4 (60 mg/kg), high dose AB4 (120 mg/kg) or DAPT $(100 \mathrm{mg} / \mathrm{kg})$ for 19 days. Xenograft tumor growth was monitored and recorded every 2 days. At the end of the experiment, the mice were humanely sacrificed by intraperitoneal injection of pentobarbital $(100 \mathrm{mg} / \mathrm{kg})$, and tumors were carefully isolated and processed for histological examination.

Immunohistochemistry (IHC). Paraformaldehyde-fixed and paraffin-embedded tissue blocks were cut into $4-\mu \mathrm{m}$ sections. The sections were dewaxed and dehydrated, followed by antigen retrieval and endogenous peroxidase blocking. IHC was performed with anti-Notch1, anti-Hes1 and anti-Ki67 antibodies (dilution, 1:100) at $4^{\circ} \mathrm{C}$ overnight. Subsequently, the slides were washed with PBS and incubated with HRP-conjugated secondary antibodies (dilution, 1:200) for $1 \mathrm{~h}$ at room temperature. Indicated proteins were visualized using a DakoEnVision Detection Kit (Dako; Agilent Technologies, Inc.). Finally, all sections were rinsed with running water, counterstained with hematoxylin and dehydrated in graded ethanol.

Statistical analysis. All analyses were performed using SPSS v20.0 software (IBM Corp.) and the results are presented as the mean \pm standard deviation. Statistically significant differences among groups were determined using one-way ANOVA and Dunnet's least significant difference post hoc tests. $\mathrm{P}<0.05$ was considered to indicate a statistically significant difference.

\section{Results}

Anticancer effect of $A B 4$ in liver cancer in vitro. To determine the potential anticancer effects of AB4 in liver cancer, two liver cancer cell lines, namely the HepG2 (hepatoblastoma) and Huh-7 (hepatocellular carcinoma) cell lines, were treated with increasing concentrations of AB4. The toxic effect of $\mathrm{AB} 4$ was evaluated using an MTT assay. As shown in Fig. 1A, $24 \mathrm{~h}$ of AB4 treatment dose-dependently inhibited HepG2 cell proliferation. $\mathrm{AB} 4$ concentrations ranging between 20 and $1,600 \mu \mathrm{mol} / 1$ readily elicited a growth inhibitory effect, and the estimated half maximal inhibitory concentration $\left(\mathrm{IC}_{50}\right)$ 

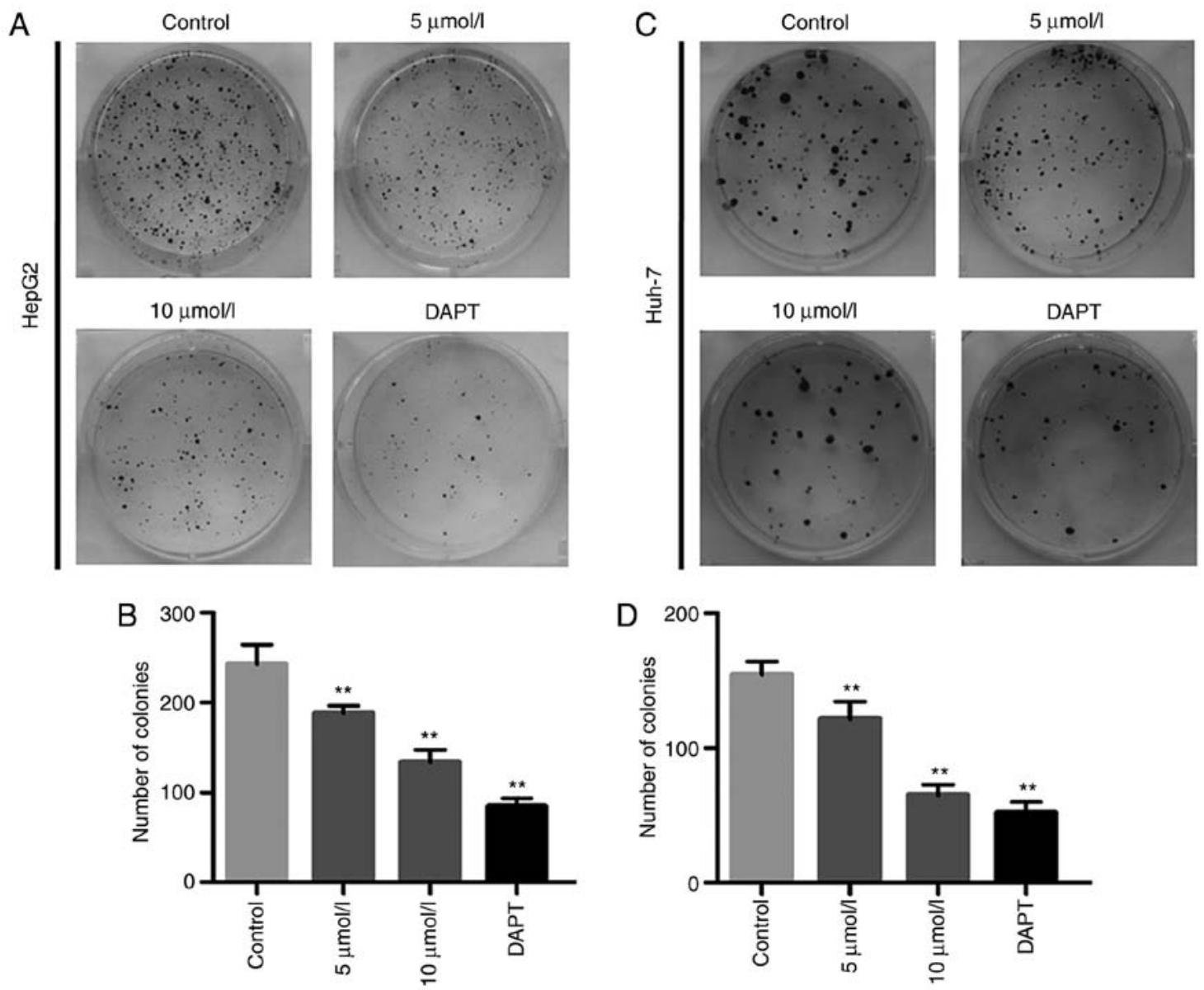

Figure 2. AB4 treatment inhibits liver cancer cell colony formation. (A and B) HepG2 and (C and D) Huh-7 cells were seeded in 6-cm culture plate and treated with the indicated low concentrations of AB4. As a positive control, cells were treated with DAPT. Cell colonies were stained and visualized with crystal violet. ${ }^{* * *} \mathrm{P}<0.01$ vs. vehicle control. AB4, an extract from the medical herb Pulsatilla chinensis (Bunge) Regel; DAPT, a $\gamma$-secretase inhibitor (GSI) that inhibits the Notch pathway.

at $24 \mathrm{~h}$ was $\sim 50 \mu \mathrm{mol} / 1$. As a positive control, treatment with $50 \mu \mathrm{mol} / 1$ DAPT, a GSI, led to $>60 \%$ cell growth inhibition. In agreement with this, AB4 also dose-dependently inhibited Huh-7 cell proliferation. The estimated $\mathrm{IC}_{50}$ of $\mathrm{AB} 4$ at $24 \mathrm{~h}$ was $\sim 45 \mu \mathrm{mol} / 1$ in Huh-7 cells (Fig. 1B).

The present study evaluated the time-dependent cytotoxicity of AB4 in both cell lines. HepG2 and Huh-7 cells were treated with AB4 at a final concentration of 50 and $45 \mu \mathrm{mol} / 1$, respectively. Treatment with $50 \mu \mathrm{mol} / \mathrm{l}$ AB4 resulted in HepG2 cell growth inhibition to a comparable magnitude of that in cells treated with $50 \mu \mathrm{mol} / 1$ DAPT. This effect peaked at $24 \mathrm{~h}$ and was maintained at a similar level at 36 and $48 \mathrm{~h}$ (Fig. 1C). Furthermore, all these aforementioned findings could also be observed in Huh-7 cells, in which treatment with $45 \mu \mathrm{mol} / 1$ AB4 time-dependently suppressed Huh-7 cell proliferation (Fig. 1D). These results revealed the anticancer effects of AB4 in liver cancer cells. Therefore, AB4 at a final concentration of 50 or $45 \mu \mathrm{mol} / 1$ was used in further experiments.

AB4 inhibits liver cancer cell colony formation. Having established that AB4 elicited cytotoxicity in liver cancer cell lines after $24 \mathrm{~h}$ of treatment, the present study next investigated whether AB4 also efficiently killed HepG2 and Huh-7 cells in experiments with relatively longer durations. Cells were seeded into $6-\mathrm{cm}$ culture plates and cell colonies were readily visualized after 2 weeks. Chronic exposure to low concentrations of
AB4 (5 and $10 \mu \mathrm{mol} / \mathrm{l})$ markedly inhibited colony formation in both cell lines. As shown in Fig. 2, chronic exposure to AB4 also dose-dependently decreased the numbers of HepG2 and Huh-7 cell colonies. Notably, the absolute colony number in the $10 \mu \mathrm{mol} / 1 \mathrm{AB} 4$ group was approximately half of that in the vehicle control group. Additionally, DAPT at a final concentration of $5 \mu \mathrm{mol} / 1$ efficiently inhibited HepG2 and Huh-7 cell colony formation. These findings demonstrated the cytotoxicity of AB4 in liver cancer cell lines and prompted the investigation of the underlying mechanism responsible for its anticancer efficacy.

AB4 induces liver cancer cell apoptosis. Extensive studies have indicated that liver cancer cells undergo apoptosis in response to various chemotherapeutic agents. Therefore, the present study investigated whether the anticancer effects of AB4 in liver cancer were dependent on the induction of cancer cell apoptosis. Following the indicated treatment, the cell apoptosis status was determined by flow cytometry. Fig. 3A shows that $24 \mathrm{~h}$ of $50 \mu \mathrm{mol} / \mathrm{l} \mathrm{AB} 4$ treatment led to $>50 \%$ of HepG2 cells undergoing apoptosis. Importantly, most of the apoptotic cells were at the early apoptotic stage. This was consistent with the notion that cytotoxic drugs primarily induce cancer cell apoptosis, rather than inducing necrosis. In agreement with this, treatment with AB4 at the same concentration and for the same treatment duration induced nearly $50 \%$ of Huh-7 

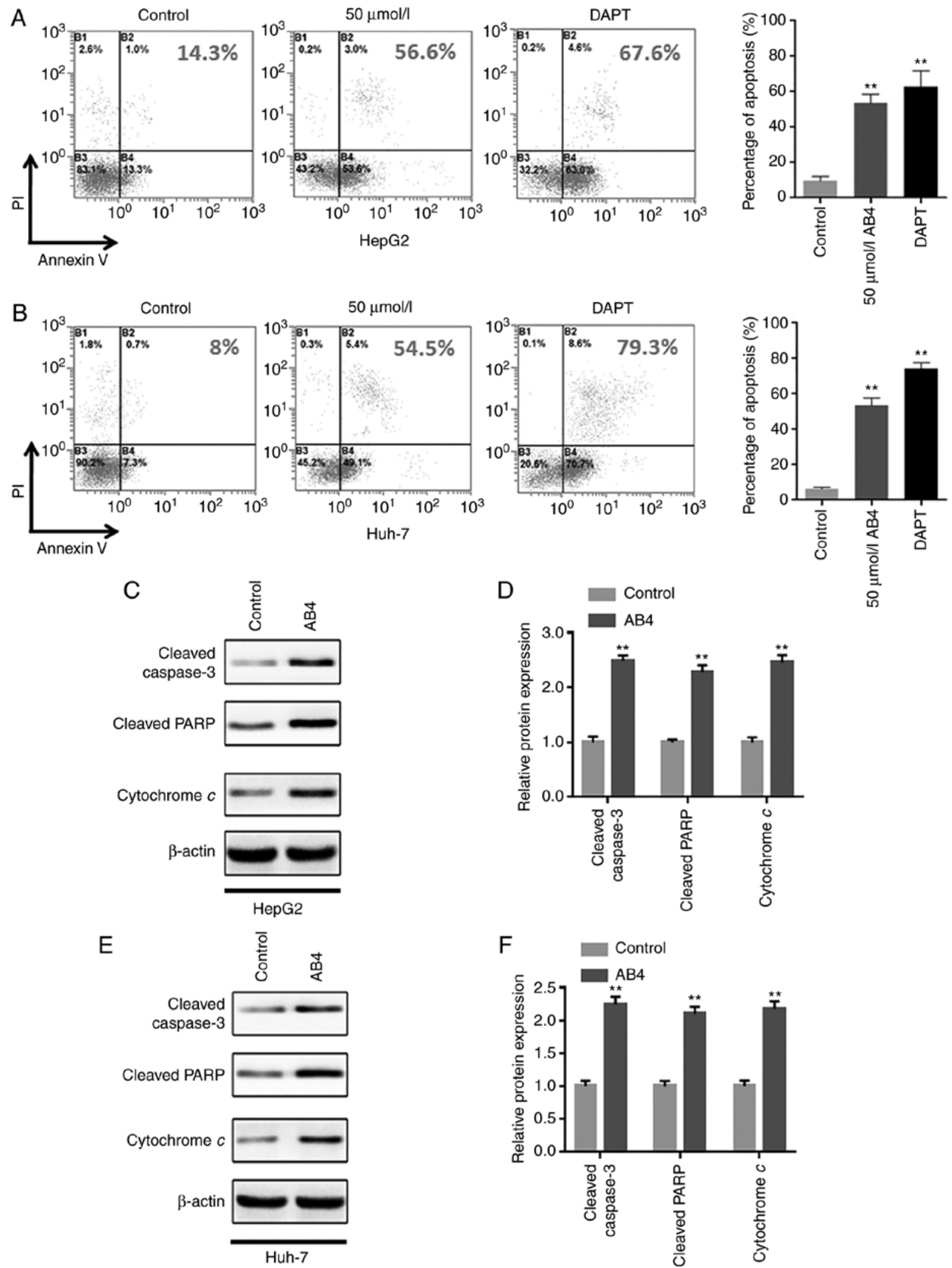

Figure 3. AB4 treatment leads to liver cancer cell apoptosis. (A) HepG2 and (B) Huh-7 cells were treated with $50 \mu \mathrm{mol} / 1 \mathrm{AB} 4 \mathrm{or}$ DAPT for $24 \mathrm{~h}$ and evaluated for cell apoptosis by flow cytometry. (C-F) After the indicated treatment, (C and D) HepG2 and (E and F) Huh-7 cells were analyzed for apoptosis by western blot analysis. Expression of apoptosis-related proteins, including cleaved caspase-3, PARP fragmentation and cytochrome $c$ release, were determined. ${ }^{* *}<0.01$ vs. vehicle control. AB4, an extract from the medical herb Pulsatilla chinensis (Bunge) Regel; DAPT, a $\gamma$-secretase inhibitor (GSI) that inhibits the Notch pathway.

cells to undergo early-stage apoptosis (Fig. 3B). Furthermore, treatment with the GSI DAPT also led to robust early-stage apoptosis in both cell lines. These compelling results indicated that apoptosis induction is a crucial mechanism of liver cancer cell death following AB4 treatment. In addition, the similar findings obtained for AB4 and DAPT indicated that both drugs may act on the same signaling pathway to induce liver cancer cell apoptosis. 

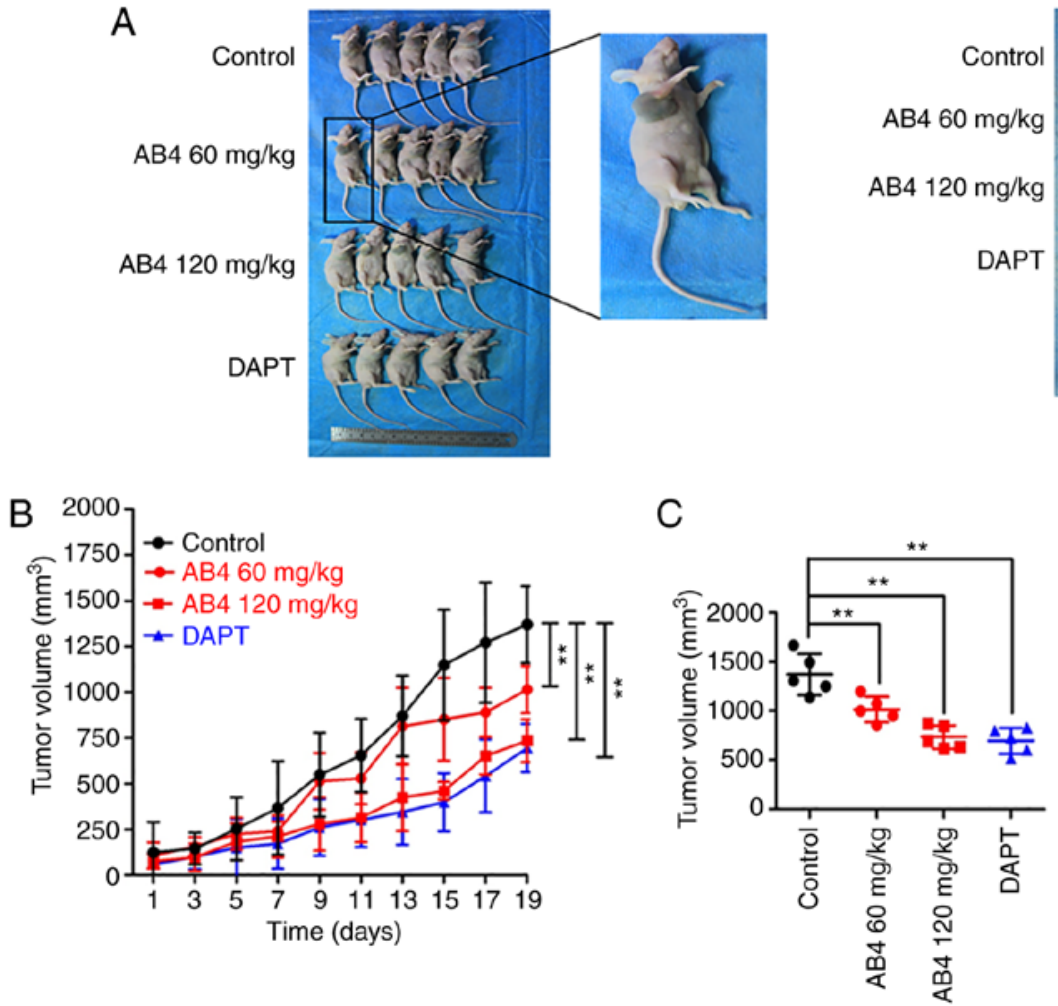
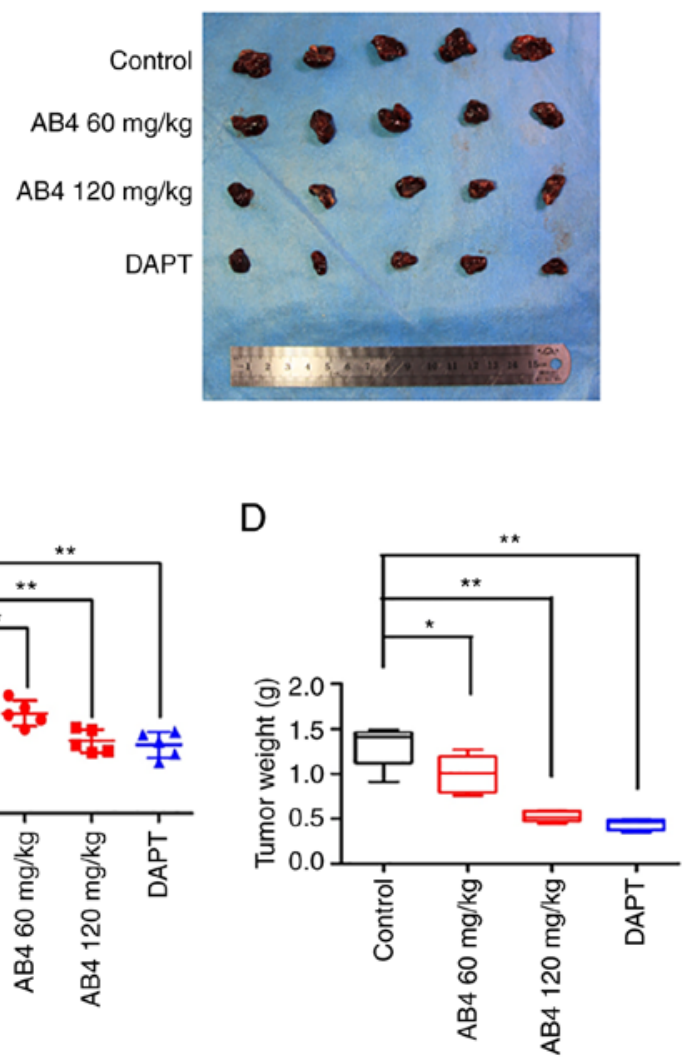

Figure 4. AB4 treatment suppresses liver cancer cell growth in vivo. (A) HepG2 cells were subcutaneously injected into null mice. The mice were treated with AB4 (60 and $120 \mathrm{mg} / \mathrm{kg}$ ) or DAPT or vehicle as a control. At the end of the experiment, the mice were humanely sacrificed and the xenograft tumors were carefully isolated. Representative images of xenograft tumors were recorded. (B) The dynamic changes in xenograft tumor volume after the indicated treatment. The black line indicates mice receiving vehicle, the red lines indicate mice receiving AB4 (circles: $60 \mathrm{mg} / \mathrm{kg}$ and squares: $120 \mathrm{mg} / \mathrm{kg}$, and the blue line indicated mice receiving DAPT. (C and D) At the end of the experiment, xenograft tumor $(\mathrm{C})$ volume and (D) weight were measured. ${ }^{*} \mathrm{P}<0.05$ and ${ }^{* *} \mathrm{P}<0.01$ vs. vehicle control. AB4, an extract from the medical herb Pulsatilla chinensis (Bunge) Regel; DAPT, a $\gamma$-secretase inhibitor (GSI) that inhibits the Notch pathway.

To validate these findings, western blot analysis was performed to evaluate the expression levels of apoptosis-related proteins following the indicated treatment. It was revealed that AB4 treatment resulted in cytochrome $c$ release, caspase-3 activation and PARP cleavage (Fig. 3C and D). Notably, the changes of the aforementioned apoptosis-related proteins were consistent in both cell lines (Fig. 3E and F). Overall, these findings indicated that $\mathrm{AB} 4$ may be a potential anticancer candidate drug for liver cancer, and acts primarily through the induction of cancer cell apoptosis.

Anticancer effect of AB4 in liver cancer in vivo. Since AB4 exhibited anticancer activity in liver cancer cell lines, the present study next determined whether this anticancer effect could also be observed in vivo. Using a xenograft model, HepG2 cells were injected into the right flank of 4-week-old male null mice. In this case, only HepG2 cells were used because this cell line rapidly grows and readily forms subcutaneous tumors in null mice. Treatment was initiated when the volume of the subcutaneous tumors reached $100 \mathrm{~mm}^{3}$. Fig. 4A shows the gross observation of subcutaneous tumors after 19 days of the indicated treatment. The dynamic change of tumor size was measured every 2 days. In Fig. 4B, it is shown that the xenograft tumors in the vehicle group markedly grew in null mice. At the end of the experiment, the average tumor volume exceeded $1,200 \mathrm{~mm}^{3}$ (maximum tumor size, 1,600 $\mathrm{mm}^{3}$; Fig. 4C). By contrast, the tumor volume gradually decreased in the AB4 group, and this effect was positively associated with the dose of AB4. To be specific, the subcutaneous tumor began to shrink on day 15 in the AB4 low dose group $(60 \mathrm{mg} / \mathrm{kg})$, whereas tumor growth began to shrink on day 9 in mice fed with a high dose of AB4 (120 mg/kg; Fig. 4C). After 19 days of the indicated treatment, the subcutaneous tumor size in the low and high dose groups was $\sim 83$ and $50 \%$ of that in the vehicle control group, respectively (Fig. 4B).

The anticancer effects of the GSI DAPT were also evaluated in vivo. Interestingly, this drug elicited profound tumor-suppressive effects in HepG2 cells in null mice (Fig. 4A-C). Notably, the $100 \mathrm{mg} / \mathrm{kg}$ DAPT regimen induced more pronounced anticancer effects. At the end of the experiment, the tumor weight in the DAPT group was $\sim 33 \%$ of that in the vehicle control group (vs. $40 \%$ in the $120 \mathrm{mg} / \mathrm{kg} \mathrm{AB} 4$ group; Fig. 4D). Both the in vitro and in vivo experiments highlighted that $\mathrm{AB} 4$ and DAPT exhibited similar anticancer efficacy in hepatocellular carcinoma (HCC), suggesting the possibility that both drugs may manipulate the same signaling pathway.

AB4 promotes liver cancer cell apoptosis in vivo. In order to investigate whether $\mathrm{AB} 4$ promotes apoptosis in vivo, the present study evaluated the histological alterations of xenograft tumors after the indicated treatment. Fig. 5A shows representative H\&E staining images of xenograft tumors from formalin-fixed paraffin-embedded tissue blocks. 
A

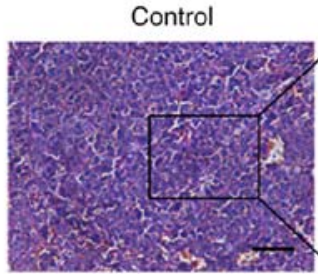

AB4 $120 \mathrm{mg} / \mathrm{kg}$

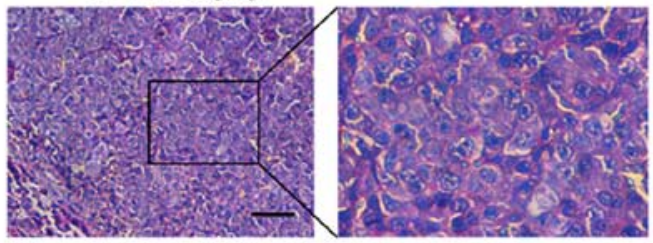

B

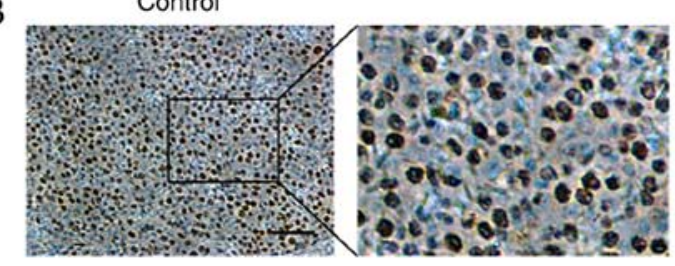

AB4 $120 \mathrm{mg} / \mathrm{kg}$

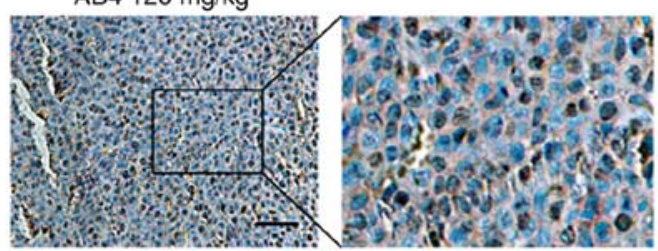

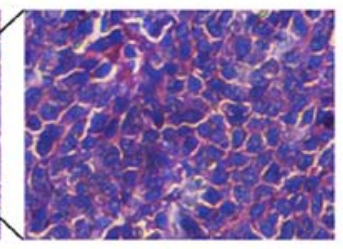

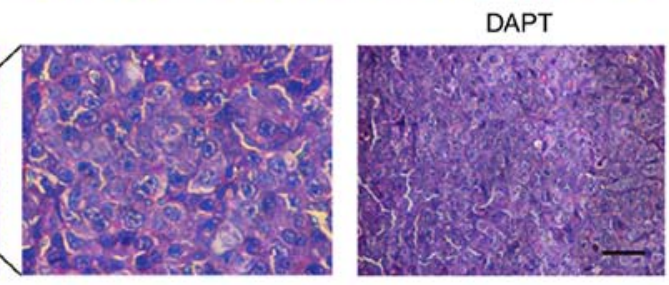

DAPT

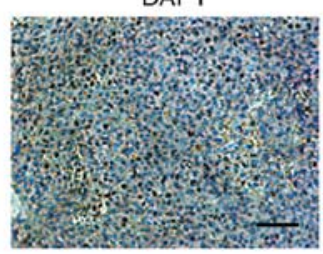

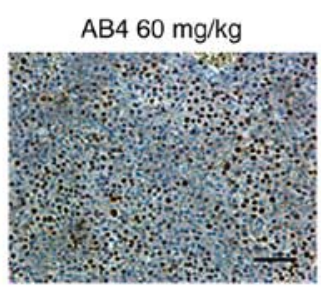

AB4 $60 \mathrm{mg} / \mathrm{kg}$

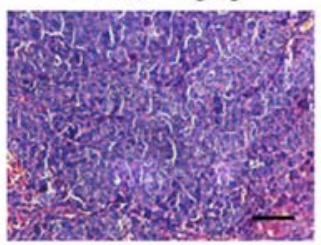

DAPT

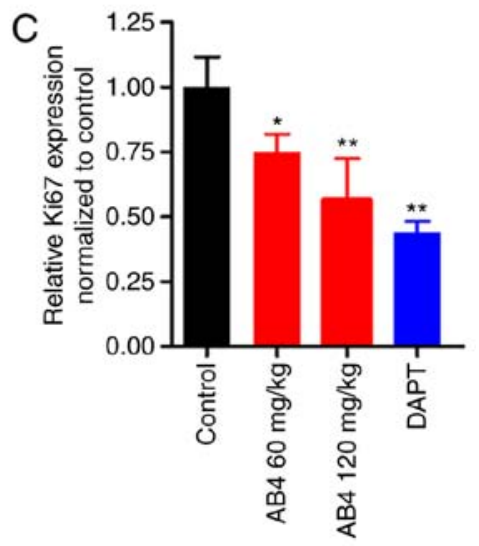

Figure 5. AB4 treatment promotes liver cancer cell apoptosis in vivo. (A) Representative histology images of the xenograft tumors. Scale bar, $50 \mu$ m. (B and C) Immunohistochemistry staining of Ki67 of the xenograft tumors. To visualize the cell nucleus, the slides were counterstained with hematoxylin. Scale bar, $50 \mu \mathrm{m}$. ${ }^{*} \mathrm{P}<0.05$ vs. vehicle control; ${ }^{*} \mathrm{P}<0.05$ and ${ }^{* *} \mathrm{P}<0.01$ vs. vehicle control. AB4, an extract from the medical herb Pulsatilla chinensis (Bunge) Regel; DAPT, a $\gamma$-secretase inhibitor (GSI) that inhibits the Notch pathway.

It was evident that $\mathrm{AB} 4$ treatment led to nucleus fragmentation, a morphological hallmark of cells undergoing apoptosis. In agreement with this, AB4 dose-dependently inhibited HCC cell proliferation, as examined by Ki67 staining, in xenograft tumors (Fig. 5B). Specifically, qualitative analysis of the Ki67 score indicated that $\mathrm{AB} 4$ at a dose of $60 \mathrm{mg} / \mathrm{kg}$ reduced the IHC score to $\sim 75 \%$ of that in the vehicle group, while this effect was more pronounced in the $120 \mathrm{mg} / \mathrm{kg}$ AB4 group. Again, DAPT produced similar anticancer effects in xenograft tumors (Fig. 5C). These data strongly suggested that AB4 is a potential anticancer drug in liver cancer in vitro and in vivo, and mainly acts through the induction of apoptosis, and AB4 and DAPT may act on the same signaling pathway.

Notch signaling as a therapeutic target of $A B 4$. The present experiments strongly indicated the anticancer effects of AB4 in liver cancer in vitro and in vivo. Additionally, the present study highlighted that inhibition of Notch signaling by the GSI DAPT exerted anticancer effects in a similar manner to AB4. These findings strongly suggested the possibility that AB4 and DAPT may act on the same signaling pathway and prompted the investigation of whether AB4 and liver cancer converged at the Notch signaling pathway.

To address these questions, the present study evaluated the effect of AB4 treatment on the expression levels of key components of the Notch signaling pathway, including Notch1, Jagged1, NICD1, Hes1 and Hey1. As illustrated in Fig. 6A, AB4 dose-dependently suppressed Notch signaling in HepG2 and Huh-7 cells, and this effect was more evident for higher doses of AB4 (50 and $100 \mu \mathrm{mol} / \mathrm{l})$. Treatment with AB4 at final concentrations of 50 and $100 \mu \mathrm{mol} / 1$ primarily inhibited Notch1 expression and, to a lesser extent, inhibited Jagged1 and NICD1 expression, suggesting that Notch1 could be the major molecular target of AB4. Therefore, the expression levels of downstream targeted proteins, such as Hes1 and Hey1, were suppressed. Consistent with these findings, the Notch1 protein was primarily expressed in the cytoplasm in xenograft tumors, whereas its expression was readily suppressed following treatment with AB4 (Fig. 6B). Furthermore, AB4 treatment inhibited Hes1 expression in vivo. Fig. 6C shows representative IHC images of Hes1 expression after the indicated treatment. In contrast to the Notch1 protein, the Hes1 protein was primarily expressed in the nucleus in xenograft tumors. However, AB4 treatment markedly reduced its expression. As a positive control, DAPT readily suppressed the protein expression of Notch1 and Hes1 in xenograft tumors. Overall, Notch signaling may be a pharmacological target of AB4. Inhibition of Notch signaling by AB4 treatment suppressed liver cancer cell proliferation and induced cancer cell apoptosis. 

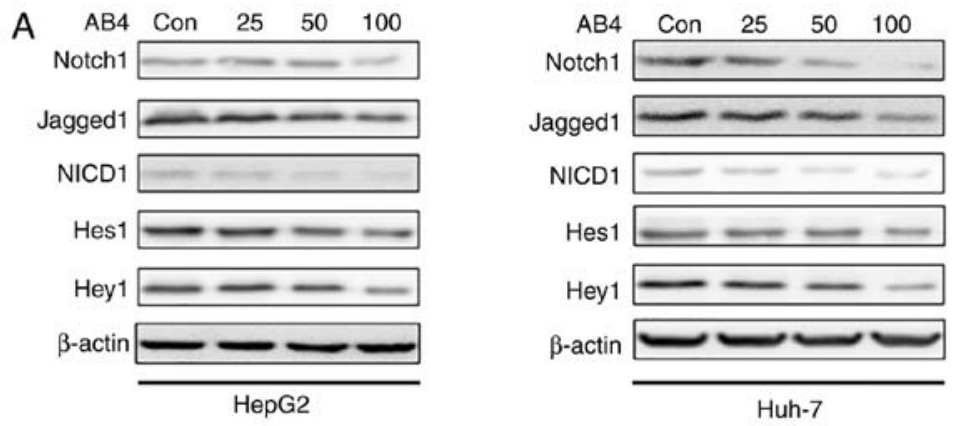

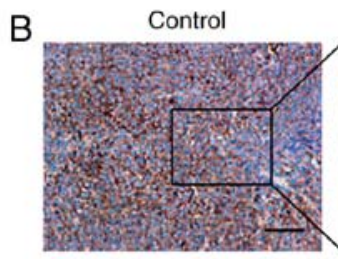

AB4 $120 \mathrm{mg} / \mathrm{kg}$

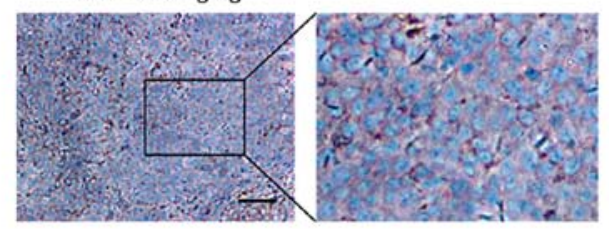

C

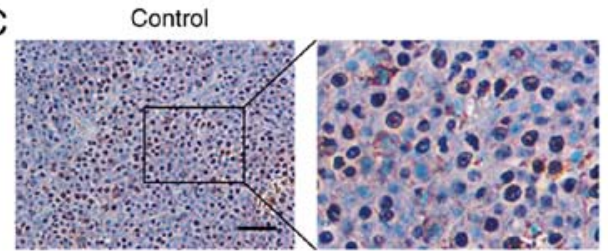

AB4 $120 \mathrm{mg} / \mathrm{kg}$

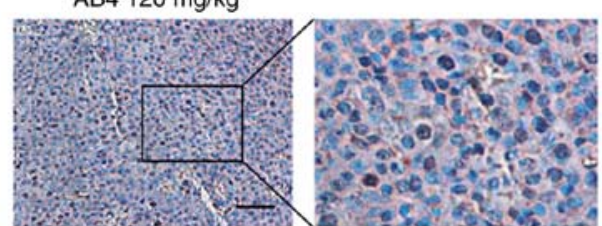

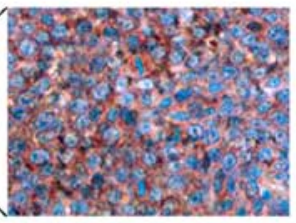

AB4 $60 \mathrm{mg} / \mathrm{kg}$

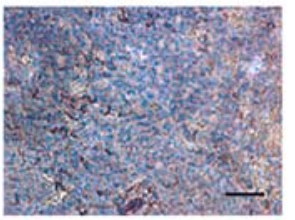

DAPT

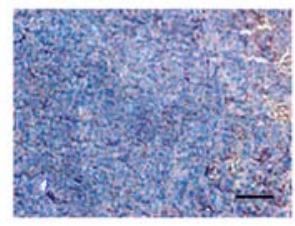

AB4 $60 \mathrm{mg} / \mathrm{kg}$

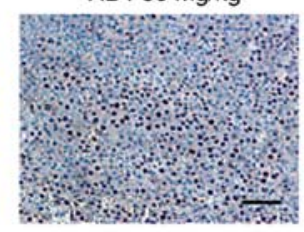

DAPT

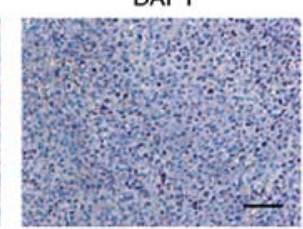

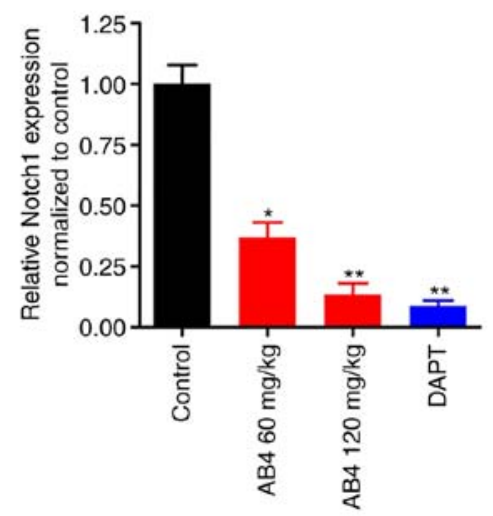

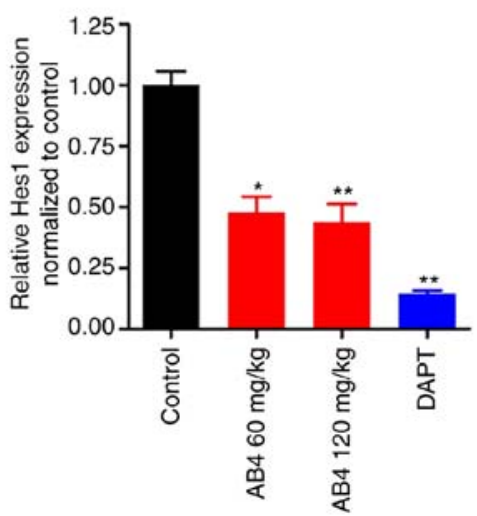

Figure 6. AB4 treatment inhibits Notch signaling. (A) Expression of Notch1 and its downstream targets in HepG2 (left panel) and Huh-7 (right panel) cells after increasing concentrations of AB4 treatment was determined by Western blot analysis. (B and C) Representative immunohistochemistry analysis of Notch1 and Hes1 expression. Scale bar, $50 \mu \mathrm{m}$. ${ }^{*} \mathrm{P}<0.05$ and ${ }^{* *} \mathrm{P}<0.01$ vs. vehicle control. AB4, an extract from the medical herb Pulsatilla chinensis (Bunge) Regel; DAPT, a $\gamma$-secretase inhibitor (GSI) that inhibits the Notch pathway; NICD1, Notch1 intracellular domain; Hes1, hes family bHLH transcription factor 1; Hey1, hes related family bHLH transcription factor with YRPW motif 1.

\section{Discussion}

The present study revealed the anticancer efficacy of a natural medicinal herb extract, AB4, an extract from the medical herb Pulsatilla chinensis (Bunge) Regel, in liver cancer in vitro and in vivo. It was revealed that AB4 readily inhibited HepG2 and Huh-7 cell proliferation and induced cancer cell apoptosis. Notably, the present study provided preliminary evidence showing that direct inhibition of Notch signaling by GSI DAPT killed both cell lines in a similar manner compared with AB4. Furthermore, it was reported that $\mathrm{AB} 4$ treatment blocked the Notch signaling pathway in vitro and in vivo. It was concluded that $\mathrm{AB} 4$ is a novel therapeutic agent for liver cancer, and it was speculated that targeting of Notch signaling underlies its anticancer activity.

Natural occurring phytochemicals have attracted increasing interest, since they are well tolerated and have broad spectrum biological activities (25). Indeed, several commercially available anticancer agents, such as taxol (26) and vincristine (27), are derived from medicinal herbs. Pulsatilla chinensis (Bunge) Regel is another paradigm of natural occurring medicinal herbs, and the bioactivities of its components have been widely investigated. Pulsatilla saponin A is the most well documented anticancer bioactive component of Pulsatilla chinensis (Bunge) Regel (14). However, this agent elicits severe cytotoxicity in untransformed cells as well. Therefore, exploring other well tolerated anticancer components is urgently necessary (16). In contrast to Pulsatilla saponin A, AB4, is well tolerable and has minimal cytotoxicity in untransformed cells. It has been widely used as a 'blood-cooling' and anti-infectious regimen in TCM (28). However, most studies have focused on the isolation, purification, pharmacokinetics and distribution 
pattern of $\mathrm{AB} 4$, and its pharmacological effect on cancer cells is largely unknown. Therefore, given the compelling clinical need for liver cancer treatment, this preliminary study was conducted to examine the anticancer efficacy of AB4.

The present study highlighted the anticancer efficacy of AB4 in the HepG2 and Huh-7 cell lines. The present study particularly focused on liver cancer because liver cancer cells are not sensitive to most cytotoxic agents. Consistently, chemotherapy fails to elicit marked and durable antitumor activity in clinical settings, and the 5-year survival of patients with advanced liver cancer is rather limited. Despite numerous successes having been achieved in the era of immunotherapy, the efficacy of immunotherapy in patients with liver cancer is far from satisfactory, and the response rate of anti-programmed cell death protein 1 (PD-1)/programmed death-ligand 1 inhibitors, including nivolumab and pembrolizumab, is $<20 \%(29,30)$. Given the pressing clinical needs, the present study reported that AB4 treatment readily suppressed liver cancer cell proliferation and resulted in apoptosis in vitro and in vivo. AB4 exhibited durable (both short-term and long-term) cytotoxicity in both cell lines and efficiently inhibited xenograft tumor outgrowth. Indeed, these pharmacological effects are interesting, suggesting AB4 as a novel candidate for liver cancer treatment. Notably, biochemical experiments have demonstrated that AB4 protects human 293 cells from platinum-induced injuries by increasing reactive oxygen species scavenger activity (31). In an adenine-induced kidney injury rat model, $\mathrm{AB} 4$ administration markedly reduces blood urea nitrogen and creatinine levels, suppresses the expression of pro-inflammatory cytokines, and attenuates collagen deposition in the renal interstitium (32). These findings highlight a kidney-protective effect of AB4 and may indicate important clinical significance. Since most cy totoxic agents are excreted through the kidney, the kidney-protective properties of AB4 could compensate chemotherapeutic agent-induced kidney injury. Therefore, it is reasonable to hypothesize that the combination of AB4 with other chemotherapeutic agents may reduce renal toxicity.

Another notable finding of the present study was the observation of the involvement of Notch signaling in the anticancer activity of AB4. The present study focused on Notch signaling because the GSI DAPT has similar anticancer efficacy compared with AB4. This finding was in compliance with the notion that Notch signaling is hyperactivated in liver cancer, thereby driving carcinogenesis and tumor progression (33). However, targeting Notch via a conventional pharmacological approach is technically challenging and severe adverse effects could occur upon non-selective inhibition of Notch (34). The present study highlighted that AB4 treatment readily inhibited Notch signaling in liver cancer in vitro and in vivo. This finding not only elucidated the mechanism underlying the anticancer efficacy of AB4, but also elicited important clinical significance. Firstly, AB4 could be considered as a novel Notch inhibitor and it was speculated that AB4 may exert broad spectrum anticancer activity, particularly in cancers driven by Notch. Secondly, a combinational strategy would result in more robust anticancer efficacy. For example, our recent study suggested that AB4 also inhibits the PI3K/Akt/mTOR signaling pathway (35). Therefore, it is reasonable to hypothesize that AB4 could be combined with chemotherapeutic agents to kill cancer cells through inhibition of multiple signaling pathways. Furthermore, the combinational strategy is expected to be well tolerated. Since AB4 possesses antioxidant and anti-inflammatory properties, combination therapy could reduce cytotoxic agent-induced hepatological and renal toxicity. In addition, increasing lines of evidence have demonstrated that Notch is a predictive biomarker for immunotherapy. Patients with non-small cell lung cancer carrying Notch mutations tend to have a higher response rate and longer progression-free survival (36). It was predicted that disrupting Notch signaling by AB4 would facilitate with immunotherapy. Therefore, it would be interesting to investigate the anticancer efficacy of AB4 and anti-PD-1 combination therapy.

In conclusion, the present study reported the anticancer efficacy of AB4 in liver cancer. AB4 treatment readily inhibited liver cancer cell proliferation and induced cell apoptosis in vitro and in vivo. Furthermore, the present study demonstrated that Notch is an important pharmacologic target for AB4. Further experiments evaluating the combinational strategy of AB4 would be interesting.

\section{Acknowledgements}

Not applicable.

\section{Funding}

This study was sponsored by grants from the National Natural Science Foundation of China (\#81703768 to QM, \#81803400 to ZX), Postdoctoral Science Foundation of China (\#2018T111161 to QM), and National Natural Science Foundation of Shandong Province (ZR2018BH045 to ZX).

\section{Availability of data and materials}

The datasets used and/or analyzed during the current study are available from the corresponding author on reasonable request.

\section{Authors' contributions}

JZ and QM designed and supervised the research. ZX and YL performed the majority of the experiments. GL, SX, XL and ZZ analyzed the data. LS, BL, YZ and TM provided technical support in conducting the experiments. All authors wrote the manuscript, read the final manuscript and approved the submission.

\section{Ethics approval and consent to participate}

All experiments were approved by the Ethic Committee of The Hospital 971 of The Navy of Chinese People's Liberation Army (Qingdao, China) and were performed in accordance with Animal Ethics Guidelines (\#401LL-2017010).

\section{Patient consent for publication}

Not applicable.

\section{Competing interests}

The authors declare that they have no competing interests. 


\section{References}

1. Bray F, Ferlay J, Soerjomataram I, Siegel RL, Torre LA and Jemal A: Global cancer statistics 2018: GLOBOCAN estimates of incidence and mortality worldwide for 36 cancers in 185 countries. CA Cancer J Clin 68: 394-424, 2018.

2. Liu X, Zhou J, Zhou N, Zhu J, Feng Y and Miao X: SYNJ2BP inhibits tumor growth and metastasis by activating DLL4 pathway in hepatocellular carcinoma. J Exp Clin Cancer Res 35: 115, 2016.

3. Han H, Wang L, Liu Y, Shi X, Zhang X, Li M and Wang T: Combination of curcuma zedoary and kelp inhibits growth and metastasis of liver cancer in vivo and in vitro via reducing endogenous $\mathrm{H}_{2} \mathrm{~S}$ levels. Food Funct 10: 224-234, 2019.

4. Wang X, Wang N, Cheung F, Lao L, Li C and Feng Y: Chinese medicines for prevention and treatment of human hepatocellular carcinoma: Current progress on pharmacological actions and mechanisms. J Integr Med 13: 142-164, 2015.

5. Wu L, Cao KX, Ni ZH, Li WD, Chen ZP, Cheng HB and Liu X: Effects of Dahuang zhechong pill on doxorubicin-resistant SMMC-7721 xenografts in mice. J Ethnopharmacol 222: 71-78, 2018.

6. Li W, Yan XT, Sun YN, Ngan TT, Shim SH and Kim YH: Anti-Inflammatory and PPAR transactivational effects of oleanane-type triterpenoid saponins from the roots of Pulsatilla koreana. Biomol Ther (Seoul) 22: 334-340, 2014.

7. Xu L, Gu L, Tao X, Xu Y, Qi Y, Yin L, Han X and Peng J: Effect of dioscin on promoting liver regeneration via activating Notch1/Jagged1 signal pathway. Phytomedicine 38: 107-117, 2018.

8. Hu Y, He K and Wang X: Role of Chinese herbal medicinal ingredients in secretion of cytokines by PCV2-induced endothelial cells. J Immunotoxicol 13: 141-147, 2016.

9. Chen Z, Duan H, Tong X, Hsu P, Han L, Morris-Natschke SL, Yang S, Liu W and Lee KH: Cytotoxicity, hemolytic toxicity, and mechanism of action of Pulsatilla Saponin D and its synthetic derivatives. J Nat Prod 81: 465-474, 2018.

10. Bang SC, Lee JH, Song GY, Kim DH, Yoon MY and Ahn BZ: Antitumor activity of Pulsatilla koreana saponins and their structure-activity relationship. Chem Pharm Bull (Tokyo) 53: $1451-1454,2005$

11. Kim Y, Bang SC, Lee JH and Ahn BZ: Pulsatilla saponin D The antitumor principle from Pulsatilla koreana. Arch Pharm Res 27: 915-918, 2004.

12. Wan JY, Zhang YZ, Yuan JB, Yang FQ, Chen Y, Zhou LD and Zhang QH: Biotransformation and metabolic profile of anemoside B4 with rat small and large intestine microflora by ultra-performance liquid chromatography-quadrupole time-of-flight tandem mass spectrometry. Biomed Chromatogr 31 : bmc.3873, 2017.

13. Zhang Y, Bao J, Wang K, Jia X, Zhang C, Huang B, Chen M, Wan JB, Su H, Wang Y and He C: Pulsatilla Saponin D inhibits autophagic flux and synergistically enhances the anticancer activity of chemotherapeutic agents against HeLa cells. Am J Chin Med 43: 1657-1670, 2015.

14. Liu Q, Chen W, Jiao Y, Hou J, Wu Q, Liu Y and Qi X: Pulsatilla saponin A, an active molecule from Pulsatilla chinensis, induces cancer cell death and inhibits tumor growth in mouse xenograft models. J Surg Res 188: 387-395, 2014.

15. Xu L, Cheng G, Lu Y and Wang S: An active molecule from Pulsatilla chinensis, Pulsatilla saponin A, induces apoptosis and inhibits tumor growth of human colon cancer cells without or with 5-FU. Oncol Lett 13: 3799-3802, 2017.

16. Tong X, Han L, Duan H, Cui Y, Feng Y, Zhu Y, Chen Z and Yang S: The derivatives of Pulsatilla saponin A, a bioactive compound from Pulsatilla chinensis: Their synthesis, cytotoxicity, haemolytic toxicity and mechanism of action. Eur J Med Chem 129: 325-336, 2017.

17. Yang Q, Bermingham NA, Finegold MJ and Zoghbi HY: Requirement of Math1 for secretory cell lineage commitment in the mouse intestine. Science 294: 2155-2158, 2001.

18. Zhu JN, Jiang L, Jiang JH, Yang X, Li XY, Zeng JX, Shi RY, Shi Y,Pan XR, Han ZP and Wei LX: Hepatocyte nuclear factor-1beta enhances the stemness of hepatocellular carcinoma cells through activation of the Notch pathway. Sci Rep 7: 4793, 2017.

19. Huang T, Zhou Y, Cheng AS, Yu J, To KF and Kang W: NOTCH receptors in gastric and other gastrointestinal cancers: Oncogenes or tumor suppressors? Mol Cancer 15: 80, 2016.
20. Takebe N, Miele L, Harris PJ, Jeong W, Bando H, Kahn M, Yang SX and Ivy SP: Targeting Notch, Hedgehog, and Wnt pathways in cancer stem cells: Clinical update. Nat Rev Clin Oncol 12: 445-464, 2015

21. Papayannidis C, DeAngelo DJ, Stock W, Huang B, Shaik MN, Cesari R, Zheng X, Reynolds JM, English PA, Ozeck M, et al: A Phase 1 study of the novel gamma-secretase inhibitor PF-03084014 in patients with T-cell acute lymphoblastic leukemia and T-cell lymphoblastic lymphoma. Blood Cancer J 5: e350, 2015.

22. Hughes DP, Kummar S and Lazar AJ: New, tolerable $\gamma$-secretase inhibitor takes desmoid down a notch. Clin Cancer Res 21: 7-9, 2015.

23. Li L, Tang P, Li S, Qin X, Yang H, Wu C and Liu Y: Notch signaling pathway networks in cancer metastasis: A new target for cancer therapy. Med Oncol 34: 180, 2017.

24. Wu CX, Xu A, Zhang CC, Olson P, Chen L, Lee TK, Cheung TT, Lo CM and Wang XQ: Notch inhibitor PF-03084014 inhibits hepatocellular carcinoma growth and metastasis via suppression of cancer stemness due to reduced activation of Notch1-Stat3. Mol Cancer Ther 16: 1531-1543, 2017.

25. Zhu PL, Fu XQ, Li JK, Tse AK, Guo H, Yin CL, Chou JY, Wang YP, Liu YX, Chen YJ, et al: Antrodia camphorata mycelia exert anti-liver cancer effects and inhibit STAT3 signaling in vitro and in vivo. Front Pharmacol 9: 1449, 2018.

26. Wang Y, Zhang C, Zhang S, Zhao Z, Wang J, Song J, Wang Y, Liu J and Hou S: Kanglaite sensitizes colorectal cancer cells to Taxol via NF- $\kappa \mathrm{B}$ inhibition and connexin 43 upregulation. Sci Rep 7: 1280, 2017.

27. Peng S, Yang K, Xu Z, Chen S and Ji Y: Vincristine and sirolimus in the treatment of kaposiform haemangioendothelioma. J Paediatr Child Health 55: 1119-1124, 2019.

28. Xu QM, Shu Z, He WJ, Chen LY, Yang SL, Yang G, Liu YL and Li XR: Antitumor activity of Pulsatilla chinensis (Bunge) Regel saponins in human liver tumor 7402 cells in vitro and in vivo. Phytomedicine 19: 293-300, 2012.

29. Zhu AX, Finn RS, Edeline J, Cattan S, Ogasawara S, Palmer D, Verslype C, Zagonel V, Fartoux L, Vogel A, et al: Pembrolizumab in patients with advanced hepatocellular carcinoma previously treated with sorafenib (KEYNOTE-224): A non-randomised, open-label phase 2 trial. Lancet Oncol 19: 940-952, 2018.

30. El-Khoueiry AB, Sangro B, Yau T, Crocenzi TS, Kudo M, Hsu C, Kim TY, Choo SP, Trojan J, Welling TH Rd, et al: Nivolumab in patients with advanced hepatocellular carcinoma (CheckMate 040): An open-label, non-comparative, phase 1/2 dose escalation and expansion trial. Lancet 389: 2492-2502, 2017.

31. He L, Zhang Y, Kang N, Wang Y, Zhang Z, Zha Z, Yang S, $\mathrm{Xu}$ Q and Liu Y: Anemoside B4 attenuates nephrotoxicity of cisplatin without reducing anti-tumor activity of cisplatin. Phytomedicine 56: 136-146, 2019.

32. Gong Q, He LL, Wang ML, Ouyang H, Gao HW, Feng YL, Yang SL, Du LJ, Li J and Luo YY: Anemoside B4 protects rat kidney from adenine-induced injury by attenuating inflammation and fibrosis and enhancing podocin and nephrin expression. Evid Based Complement Alternat Med 2019: 8031039, 2019.

33. Villanueva A, Alsinet C, Yanger K, Hoshida Y, Zong Y, Toffanin S, Rodriguez-Carunchio L, Solé M, Thung S, Stanger BZ and Llovet JM: Notch signaling is activated in human hepatocellular carcinoma and induces tumor formation in mice. Gastroenterology 143: 1660-1669.e7, 2012.

34. Rizzo P, Mele D, Caliceti C, Pannella M, Fortini C, Clementz AG, Morelli MB, Aquila G, Ameri P and Ferrari R: The role of notch in the cardiovascular system: Potential adverse effects of investigational notch inhibitors. Front Oncol 4: 384, 2015.

35. Xue S, Zhou Y, Zhang J, Xiang Z, Liu Y, Miao T, Liu G, Liu B, Liu X, Shen L, et al: Anemoside B4 exerts anti-cancer effect by inducing apoptosis and autophagy through inhibiton of PI3K/Akt/mTOR pathway in hepatocellular carcinoma. Am J Transl Res 11: 2580-2589, 2019.

36. Zhang K, Hong X, Song Z, Xu Y, Li C, Wang G, Zhang Y, Zhao X, Zhao Z, Zhao J, et al: Identification of deleterious NOTCH mutation as novel predictor to efficacious immunotherapy in NSCLC. Clin Cancer Res 26: 3649-3661, 2020.

This work is licensed under a Creative Commons Attribution-NonCommercial-NoDerivatives 4.0 International (CC BY-NC-ND 4.0) License. 